\title{
Substitution site and effects on magnetism in Sr-for-Ca substituted $\mathrm{CaBaCo}_{4} \mathrm{O}_{7}$
}

G. Aurelio, ${ }^{1}$ J. Curiale, ${ }^{1,2}$ F. Bardelli, ${ }^{3}$ R. Junqueira Prado, ${ }^{4}$ L. Hennet, ${ }^{5}$ G. Cuello, ${ }^{6}$ J. Campo, ${ }^{7}$ and D. Thiaudière ${ }^{8}$

1) Consejo Nacional de Investigaciones Cientificas y Técnicas, Centro Atómico Bariloche - Comisión Nacional de Energía Atómica, 8400 S. C. de Bariloche, RN, Argentina ${ }^{\mathrm{a}}$

${ }^{2)}$ Instituto Balseiro, Universidad Nacional de Cuyo, 8400 S. C. de Bariloche, RN, Argentina.

3) Institut des Sciences de la Terre, Maison des Geosciences, Grenoble, France

4) Instituto de Física, Universidade Federal de Mato Grosso, 78060-900 Cuiabá-MT, Brazil

5) Conditions Extrêmes et Matériaux : Haute Température et Irradiation, 45071 Orléans, France

6) Institut Laue Langevin, F-38042 Grenoble, France

7) Instituto de Ciencia de Materiales de Aragón, Facultad de Ciencias, Universidad de Zaragoza-CSIC, 50009 Zaragoza, Spain

8) Synchrotron SOLEIL, 91192 Gif sur Yvette, France

(Dated: 26 August 2015) 
Cationic substitutions in the novel magnetoelectric compound $\mathrm{CaBaCo}_{4} \mathrm{O}_{7}$ lead to profound changes in its magnetic and electric behavior. In this work we present a structural study of the isovalent substitution Sr-for-Ca in $\mathrm{CaBaCo}_{4} \mathrm{O}_{7}$. X-ray as well as neutron powder diffraction experiments are reported for a series of samples $\mathrm{Ca}_{1-x} \mathrm{Sr}_{x} \mathrm{BaCo}_{4} \mathrm{O}_{7}$ with $0 \leq x \leq 0.10$. Special emphasis is given to the identification of the substitution site, as $\mathrm{Sr}$ has also been reported to substitute for Ba in this crystal structure. The solubility limit for $\mathrm{Sr}$ at the Ca site is shown to be at $x \simeq 0.08$. The variation of lattice constants with Sr-doping firmly supports the Sr-for-Ca substitution. Rietveld refinements of the Sr-substituted samples are presented, and used as starting point to analyse the local structure around Sr by means of X-ray absorption spectroscopy at the Sr K-edge. Both the near-edge absorption as the extended absorption fine-structure confirm the substitution of $\mathrm{Sr}$ for $\mathrm{Ca}$, giving definite support to the proposed nominal formula. In addition, macroscopic magnetization measurements are presented which reveal the striking effects of Sr-substitution over the magnetic landscape of this puzzling compound.

\footnotetext{
a)Electronic mail: gaurelio@cab.cnea.gov.ar
} 


\section{INTRODUCTION}

During the last decade, a novel member of the cobalt-rich oxides family has gained increasing attention. The so-called "114" -cobaltate $R \mathrm{BaCo}_{4} \mathrm{O}_{7}$ was discovered in 2002 for $R=\mathrm{Y}^{1}$ and, since then, synthesized with other lanthanides. This compound displays at least two very attractive features. First, it offers the possibility to study frustrated magnetism in the unique topology of a three-dimensional framework of Co tetrahedra in interconnected triangular and Kagomé lattices. Geometric frustration is related to intensively studied phenomena in spin ices and multiferroic materials ${ }^{2,3}$; and this compound opens a new possible scenario for its study. Second, it shows an outstanding capacity of reversible absorption and desorption of gases, which turns these cobaltates promising candidates for oxygen-storage and gas-sensors applications ${ }^{4,5}$.

In 2009, Caignaert et al. ${ }^{6}$ synthesized this compound for $R=\mathrm{Ca}$ which triggered even more exciting features, as the Ca-member, by changing the mixed valence state of $\mathrm{Co}$, the structure, and the charge order, dramatically varies the magnetic and electric properties. The $\mathrm{CaBaCo}_{4} \mathrm{O}_{7}$ compound was shown to become ferrimagnetic below $60 \mathrm{~K}^{7,8}$ with a complex non-collinear arrangement of spins, and it was later discovered that it concomitantly displays a dramatic increase of electric polarization of $17000 \mu \mathrm{C} / \mathrm{cm}^{2}$, driven by exchange-striction ${ }^{9,10}$. Whereas it was initially thought that the compound was ferroelectric ${ }^{11}$, it is rather defined as a ferromagnetic pyroelectric, as the polarization cannot be switched by an external electric field ${ }^{10,12}$. These very recent findings open up the route to new magnetoelectric and multiferroic materials in which the structural properties play a major role.

In this work, we aim at studying the effect of an isovalent substitution at the Ca site by replacing $\mathrm{Ca}^{2+}$ with $\mathrm{Sr}^{2+}$. Other cationic substitutions at the 
Co and $\mathrm{Ba}$ sites have shown to affect the magnetic properties ${ }^{13-15}$. This paper focuses in the room-temperature structural aspects of the Sr-substitution, given the importance of correctly identifying the substitution sites in the structure ${ }^{16}$. To this end, we have performed not only diffraction experiments using X-rays and neutrons, but also studied the local environment of Sr atoms by means of X-Ray absorption spectroscopy. Finally, we analyse the striking effects that this substitution has on the magnetic properties, reporting macroscopic dc and acmagnetization measurements as a function of temperature and frequency.

\section{EXPERIMENTAL METHODS}

\section{A. Synthesis}

Polycrystalline samples of nominal $\mathrm{CaBaCo}_{4} \mathrm{O}_{7}$ (hereafter called $\mathrm{Sr} 0 \%$ ) and $\mathrm{Ca}_{1-x} \mathrm{Sr}_{x} \mathrm{BaCo}_{4} \mathrm{O}_{7}$ with $x=0.02(\mathrm{Sr} 2 \%), x=0.05(\mathrm{Sr} 5 \%), x=0.07(\mathrm{Sr} 7 \%)$ and $x=0.10(\mathrm{Sr} 10 \%)$ were prepared by conventional solid-state reaction. High-purity powders of previously dried $\mathrm{Y}_{2} \mathrm{O}_{3}, \mathrm{BaCO}_{3}, \mathrm{SrCO}_{3}$ and $\mathrm{Co}_{3} \mathrm{O}_{4}$ were throughly mixed in an agate mortar at stoichiometric weights. After a de-carbonation process at $1173 \mathrm{~K}$ for $12 \mathrm{~h}$, the mixtures were pressed into pellets. Pellets were annealed during $12 \mathrm{~h}$ to $15 \mathrm{~h}$ at $1373 \mathrm{~K}$ and cooled at $3 \mathrm{~K} / \mathrm{min}$ in the furnace. After a regrinding, the compression and annealing at $1373 \mathrm{~K}$ in air were repeated and as a final step samples were quenched from $1373 \mathrm{~K}$ to L- $\mathrm{N}_{2}$ to obtain the stoichiometric compound $^{6,8}$. 


\section{B. X-ray diffraction}

Room-temperature X-ray powder diffraction (XRPD) data were collected using a PANalytical Empyrean diffractometer with $\mathrm{Cu}-\mathrm{K}_{\alpha}$ radiation to check the phase purity. A small amount of a secondary phase was detected for sample Sr10\%, which was identified as a perovskite belonging to the $(\mathrm{Sr}, \mathrm{Ba}) \mathrm{CoO}_{3-\delta}$ family, most likely $\mathrm{Sr}_{0.2} \mathrm{Ba}_{0.8} \mathrm{CoO}_{2.3}$ (reference code ICSD 260871). We relate this to the solubility limit for Sr, as we discuss later in the manuscript.

\section{Neutron diffraction}

High-resolution neutron powder diffraction (NPD) experiments were performed at the Institut Laue-Langevin (ILL) in Grenoble, France. NPD data at room temperature were collected in the D2B diffractometer for samples $\mathrm{Sr} 2 \%$

and Sr5\%. A wavelength of $\sim 1.59 \AA$ was used, with an angular span of $150^{\circ}$ and a step of $0.05^{\circ}$. The collimated beam on the sample was of $32 \mathrm{~mm} \times 12 \mathrm{~mm}$. A $\mathrm{NaCaAlF}$ standard was used to calibrate the neutrons wavelength, yielding the value $\lambda=1.5943 \pm 0.0001 \AA$. Measurements were performed in vanadium cylinders of $6 \mathrm{~mm}$ diameter and $8 \mathrm{~cm}$ height filled with sample, with a collection time of $4 \mathrm{~h}$. Data were processed with the LAMP software $^{17}$ and the diffractograms thus obtained were refined with the full-pattern analysis Rietveld method, using the program FULLPROF ${ }^{18}$.

\section{XAS}

X-ray absorption spectroscopy (XAS) is particularly powerful to determine the substitution site of dopants, because it is an element-selective tool, allowing to 
study the local environment of selected atomic species. Extended X-ray absorption fine structure (EXAFS) spectra at the Sr K-edge (16105 eV) were collected in fluorescence mode at the DIFFABS beamline of the Soleil synchrotron (France). EXAFS spectra were acquired with a step of $1 \mathrm{eV}$ in the near edge region (16 to $16.4 \mathrm{keV}$ ), and $2 \mathrm{eV}$ in the EXAFS region (up to $16.8 \mathrm{keV}$ ). The acquisition was done using an 4-element silicon drift detector. A double-crystal monochromator equipped with two silicon (111) crystals was used. Energy was calibrated using a $25 \mu \mathrm{m}$ Y foil (K edge : $17038 \mathrm{eV}$ ). Three scans were collected at room temperature and ambient pressure for a total acquisition time of $3 \mathrm{~h}$.

XANES spectra at the Sr K-edge were simulated using the ab-initio FEFF8.4 code $^{19}$. The full multiple scattering (FMS) and self-consistent-field (SCF) atomic potential calculation routines were found to be crucial to obtain good simulations. The Hedin-Lundqvist exchange potential ${ }^{20}$ was used. For the calculation of the SCF muffin-tin atomic potential a cluster of size $7.2 \AA$ (113 atoms) was used. A cluster of 300 atoms was used for the FMS calculation. The theoretical XANES spectra were shifted in order to match the experimental edge energy.

Standard procedures were followed to extract the EXAFS signal ${ }^{21}$, i.e.: subtraction of the pre-edge background, normalization, alignment and energy calibration, and spline modeling of the post-edge atomic background. The EXAFS signal was Fourier filtered to exclude contributions of noise and coordination shells above the third one. The extraction of the EXAFS signal and the structural refinements were performed using the ESTRA-FITEXA software package ${ }^{22}$. Minimizations were conducted in the wave vector space $(k)$ to avoid possible artifacts introduced by Fourier filtering. The number of free parameters in the fits never exceeded the number of independent points $\left(\mathrm{N}_{\text {ind }}=(2 \Delta k \cdot \Delta R) / \pi+1\right)$. The amplitude and phase photoelectron functions were calculated ab-initio using the FEFF8 code $^{19}$. The crystallographic clusters centered on the absorption specie (Sr) used 
for FEFF calculations were obtained from the structure refined from neutron diffraction data, as described in the following.

\section{E. Magnetization measurements}

DC magnetization measurements were performed using two superconducting quantum interference devices (SQUID, Quantum Design, San Diego, USA) magnetometers at low temperature $(5 \mathrm{~K}<T<350 \mathrm{~K})$ under different applied magnetic fields between $1 \mathrm{kOe}$ and $140 \mathrm{kOe}$, at warming/cooling rates of $2 \mathrm{~K} / \mathrm{min}$. The acsusceptibility was measured using a PPMS (Quantum Design, San Diego, USA) with frequencies ranging from $1 \mathrm{~Hz}$ to $10 \mathrm{kHz}$ with $H_{a c}=10$ Oe and $H_{d c}=0$ Oe. For all the magnetization experiments, small fragments of the sintered ceramic pellets were used.

\section{RESULTS}

\section{A. Room-temperature structure}

Rietveld refinements of the NPD room-temperature data of samples $\mathrm{Sr} 2 \%$ and Sr5\% are presented in Fig. 1. Structural data obtained from the refinement are summarized in Table I. The refinements return a structural model in the Pbn $2_{1}$ space group, in line with that reported for the parent compound ${ }^{7}$. Additional trials explicitly including $\mathrm{Sr}$ at the nominal $\mathrm{Ca}$ site and/or at the Ba site, did not improve the results significantly. No unexplained reflections are observed in these samples, indicating that Sr has not been segregated in a secondary phase within the limits of detection of NPD for these samples. The occupation of oxygen sites refined to full occupancy within the uncertainty limits, in agreement with the 
stoichiometric formula expected from the synthesis protocol used in preparing the samples.

The most relevant bond-lengths are summarized in Tables II and III for samples $\mathrm{Sr} 2 \%$ and $\mathrm{Sr} 5 \%$, together with the results of bond-valence-sum (BVS) calculations performed using the KDist software ${ }^{23}$. Negligible differences in the interatomic distances are observed between samples $\mathrm{Sr} 2 \%$ and $\mathrm{Sr} 5 \%$. It is interesting to compare these data with the interatomic distances in the parent compound $\mathrm{CaBaCo}_{4} \mathrm{O}_{7}$. The room-temperature structure for $\mathrm{CaBaCo}_{4} \mathrm{O}_{7}$ was only reported by Caignaert et al. ${ }^{6}$. Using their atomic positions, the calculated bond-lengths are slightly different to ours. Other reports of the lowtemperature structure of $\mathrm{CaBaCo}_{4} \mathrm{O}_{7}{ }^{7}$ and the room-temperature structure of Zn-doped $\mathrm{CaBaCo}_{4-x} \mathrm{Zn}_{x} \mathrm{O}_{7}{ }^{24}$, are on the other hand in full agreement with our results. The discrepancy with the reported room-temperature structure for $\mathrm{CaBaCo}_{4} \mathrm{O}_{7}$ in Ref. ${ }^{6}$ may be due to the fact that the latter is based on laboratory XRD data, whereas NPD (used here as well as in Refs. ${ }^{7}$ and $^{24}$ ) yields more reliable positions for the oxygen atoms. Based on these considerations, we propose that the structural model obtained in this work for Sr-doped samples is better for the description of $\mathrm{CaBaCo}_{4} \mathrm{O}_{7}$ at room-temperature.

The interatomic distances and BVS reported in Tables II and III also confirm that the Sr-substitution does not disrupt the scheme of charge order between $\mathrm{Co}^{2+}$ at $\mathrm{Co} 2$ and $\mathrm{Co} 3$ sites, and the hybridized $\mathrm{Co}^{3+} / \mathrm{Co}^{2+}\left(3 d^{7} \underline{\mathrm{L}}\right)$ at sites $\mathrm{Co} 1$ and $\mathrm{Co} 4$ proposed for the parent compound ${ }^{7}$.

The effects of the Sr substitution over the lattice parameters at room temperature are presented in Fig. 2, as well as on the volume of the cell normalized per unit formula. An anisotropic distortion is observed with the increase of Srsubstitution, while the $c$ parameter remains unchanged (within experimental uncertainty). Most importantly, there is an increase in the unit cell volume. This is 

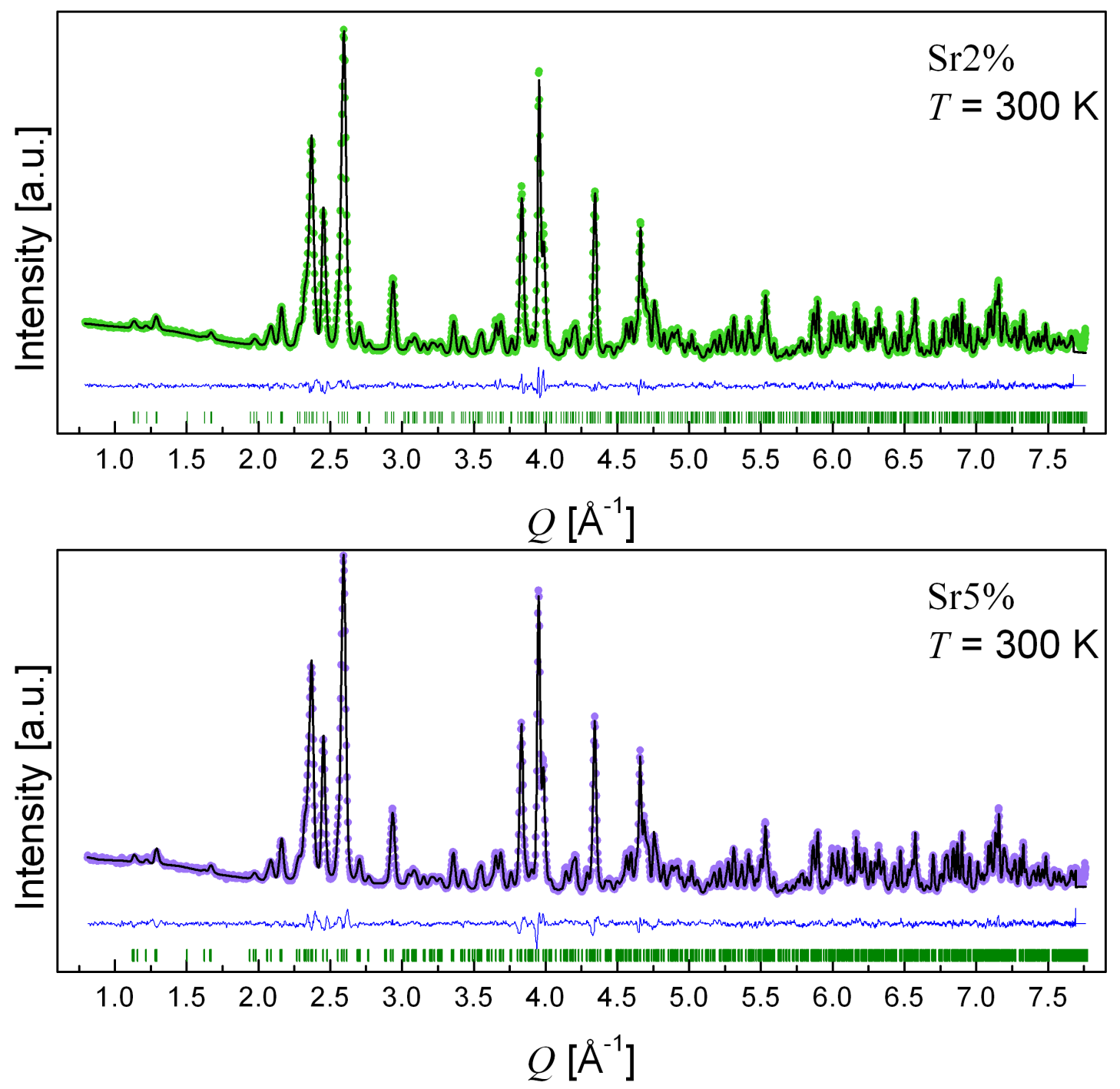

FIG. 1. Rietveld refinement for samples Sr2\% and Sr5\% from NPD data collected at room temperature in the high-resolution diffractometer D2B at ILL. Vertical bars at the bottom indicate Bragg reflections from the orthorhombic $P b n 2_{1}$-phase. The line at the bottom corresponds to the difference between the experimental and calculated patterns. 


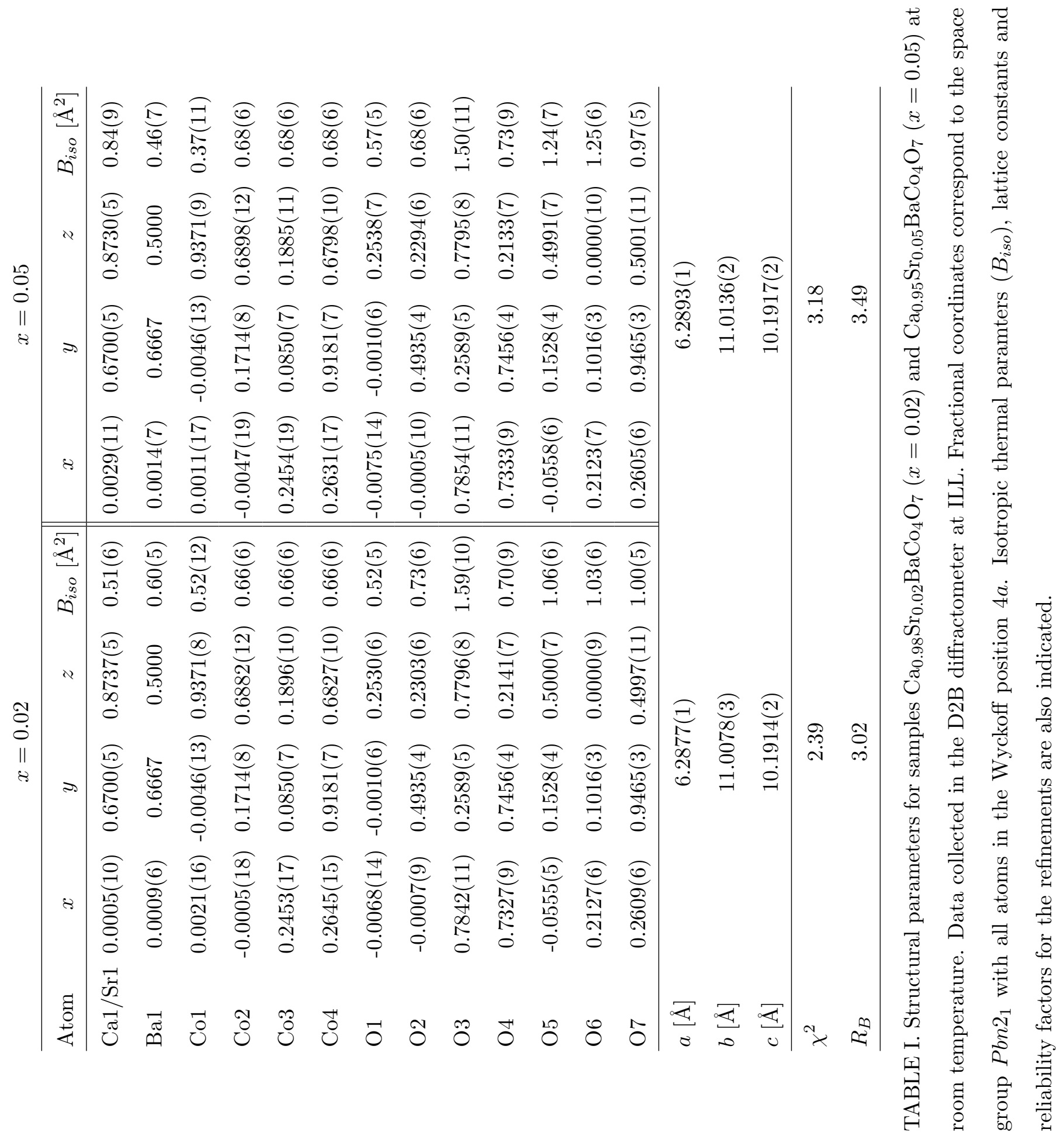




\begin{tabular}{|c|c|c|c|c|c|c|}
\hline & \multicolumn{3}{|c|}{$x=0.02$} & \multicolumn{3}{|c|}{$x=0.05$} \\
\hline \multirow[t]{2}{*}{$\mathrm{Co}-\mathrm{O}$} & Bond-length & Average value & BVS & Bond-length & Average value & BVS \\
\hline & {$[\AA]$} & {$[\AA]$} & & {$[\AA]$} & {$[\AA]$} & \\
\hline Co1-O5 & $1.785(14)$ & $1.856(12)$ & 2.66 & $1.798(14)$ & $1.856(13)$ & 2.63 \\
\hline Co1-O1 & $1.877(10)$ & & & $1.869(12)$ & & \\
\hline Co1-O6 & $1.878(13)$ & & & $1.882(13)$ & & \\
\hline $\mathrm{Co} 1-\mathrm{O} 7$ & $1.884(12)$ & & & $1.874(12)$ & & \\
\hline $\mathrm{Co} 2-\mathrm{O} 3$ & $1.904(13)$ & $1.947(13)$ & 2.02 & $1.867(13)$ & $1.952(13)$ & 1.99 \\
\hline $\mathrm{Co} 2-\mathrm{O} 4$ & 1.934(12) & & & $1.952(13)$ & & \\
\hline $\mathrm{Co} 2-\mathrm{O} 5$ & $1.960(14)$ & & & $1.981(14)$ & & \\
\hline $\mathrm{Co} 2-\mathrm{O} 1$ & $1.989(11)$ & & & $2.008(11)$ & & \\
\hline $\mathrm{Co} 3-\mathrm{O} 2$ & $1.941(11)$ & $1.953(12)$ & 1.99 & $1.937(13)$ & $1.954(13)$ & 1.97 \\
\hline Co3-O6 & $1.952(14)$ & & & $1.943(15)$ & & \\
\hline Co3-O1 & $1.957(13)$ & & & $1.957(14)$ & & \\
\hline Co3-O3 & $1.962(10)$ & & & $1.980(12)$ & & \\
\hline $\mathrm{Co} 4-\mathrm{O} 2$ & $1.834(11)$ & $1.890(12)$ & 2.39 & $1.859(11)$ & $1.884(12)$ & 2.41 \\
\hline $\mathrm{Co} 4-\mathrm{O} 4$ & $1.841(9)$ & & & $1.817(9)$ & & \\
\hline $\mathrm{Co} 4-\mathrm{O} 7$ & $1.891(15)$ & & & $1.861(15)$ & & \\
\hline $\mathrm{Co} 4-\mathrm{O} 1$ & $1.993(12)$ & & & $2.000(13)$ & & \\
\hline
\end{tabular}

TABLE II. Cobalt-oxygen bond lengths determined at room-temperature from the Rietveld refinement of D2B data, for samples $\mathrm{Ca}_{0.98} \mathrm{Sr}_{0.02} \mathrm{BaCo}_{4} \mathrm{O}_{7}$ and $\mathrm{Ca}_{0.95} \mathrm{Sr}_{0.05} \mathrm{BaCo}_{4} \mathrm{O}_{7}$. The bond-valence-sums (BVS), indicative of the cobalt oxidation states, are also shown.

already pointing that $\mathrm{Sr}$ is substituting for $\mathrm{Ca}$, a smaller cation. It is interesting to compare the behavior of our samples with those of $\mathrm{Ca}\left(\mathrm{Ba}_{1-x} \mathrm{Sr}_{x}\right) \mathrm{Co}_{4} \mathrm{O}_{7}$ reported 


\begin{tabular}{|c|c|c|c|c|c|c|}
\hline & \multicolumn{3}{|c|}{$x=0.02$} & \multicolumn{3}{|c|}{$x=0.05$} \\
\hline \multirow[t]{2}{*}{$\mathrm{M}-\mathrm{O}$} & Bond-length & Average value & BVS & Bond-lengtl & Average valu & BVS \\
\hline & {$[\AA]$} & {$[\AA]$} & & {$[\AA]$} & {$[\AA]$} & \\
\hline Ca1-O3 & $2.254(9)$ & $2.335(8)$ & 2.07 & $2.263(10)$ & $2.338(9)$ & 2.04 \\
\hline $\mathrm{Ca} 1-\mathrm{O} 2$ & $2.318(7)$ & & & $2.328(8)$ & & \\
\hline Ca1-O6 & $2.340(8)$ & & & $2.339(9)$ & & \\
\hline Ca1-O7 & $2.359(9)$ & & & $2.392(9)$ & & \\
\hline $\mathrm{Ca} 1-\mathrm{O} 5$ & $2.362(8)$ & & & $2.336(8)$ & & \\
\hline Ca1-O4 & $2.375(8)$ & & & $2.368(9)$ & & \\
\hline Ba1-O3 & $2.749(8)$ & $3.150(5)$ & 1.59 & $2.746(8)$ & $3.151(7)$ & 1.55 \\
\hline $\mathrm{Ba} 1-\mathrm{O} 4$ & $2.799(6)$ & & & $2.802(7)$ & & \\
\hline Ba1-O5 & $2.799(3)$ & & & $2.805(6)$ & & \\
\hline $\mathrm{Ba} 1-\mathrm{O} 7$ & $2.852(3)$ & & & $2.831(6)$ & & \\
\hline Ba1-O6 & $2.880(3)$ & & & $2.893(6)$ & & \\
\hline Ba1-O2 & $2.936(6)$ & & & $2.914(6)$ & & \\
\hline $\mathrm{Ba} 1-\mathrm{O} 2$ & $3.345(6)$ & & & $3.350(6)$ & & \\
\hline Ba1-O6 & $3.462(3)$ & & & $3.461(6)$ & & \\
\hline $\mathrm{Ba} 1-\mathrm{O} 4$ & $3.474(7)$ & & & $3.483(7)$ & & \\
\hline Ba1-O7 & $3.490(3)$ & & & $3.506(6)$ & & \\
\hline Ba1-O5 & $3.496(3)$ & & & $3.489(6)$ & & \\
\hline Ba1-O3 & $3.513(8)$ & & & $3.527(8)$ & & \\
\hline
\end{tabular}

TABLE III. Calcium and barium nearest neighbors bond-lengths determined at room-temperature from the Rietveld refinement of D2B data, for samples $\mathrm{Ca}_{0.98} \mathrm{Sr}_{0.02} \mathrm{BaCo}_{4} \mathrm{O}_{7}$ and $\mathrm{Ca}_{0.95} \mathrm{Sr}_{0.05} \mathrm{BaCo}_{4} \mathrm{O}_{7}$. The bond-valence-sums (BVS), indicative of the cation's oxidation state, are also shown. 
recently by Motin Seikh et al. ${ }^{25}$. The latter, in contrast to ours, show a decrease of the unit cell volume with the Sr-for-Ba substitution, which is consistent with the smaller size of $\mathrm{Sr}^{+2}$ with respect to $\mathrm{Ba}^{+2}$ (light circles in Fig. 2). Apparently, the structure is flexible enough to admit both substitutions, which makes it very important to ensure the correct substitution site before drawing any conclusions over the physical properties. To that end, a XAS study was conducted at the Sr $\mathrm{K}$-edge and is presented below.

\section{B. Solubility limit}

The presence of a segregated phase in the Sr10\% sample $\left(x_{S r}=0.10\right)$ was first suggested by our XRPD measurements. Diffractograms at room-temperature for the $\mathrm{Sr} 0 \%$ and Sr10\% samples are shown in Fig. 3 (a) and (b), respectively, collected using a laboratory X-ray diffractometer. A shoulder to the right of the $\left(\begin{array}{lll}0 & 2 & 3\end{array}\right)$ (1 113 ) reflections located at $31^{\circ}$ suggests the presence of an additional phase in Sr10\% (b). This was confirmed in a synchrotron XRPD experiment performed at the LNLS in Brazil with a much better resolution ${ }^{26}$. In Fig. 3(c) and (d) we show the same $Q$-range in the LNLS diffractograms collected at $15 \mathrm{~K}$. The presence of a segregated phase in the Sr10\% sample is clearly visible (indicated with an arrow in Fig. 3(d)), while it is absent in the Sr7\% sample, confirming that the solubility limit for Sr at the Ca site lies between 7 and 10 at.\% Sr-doping. Although the remaining reflections from the segregated phase are quite weak (not shown in the figure), the phase may be assigned to a perovskite belonging to the family $\mathrm{Sr}_{x} \mathrm{Ba}_{1-x} \mathrm{CoO}_{2.5}$, most likely with $x \sim 0.2^{27,28}$. 


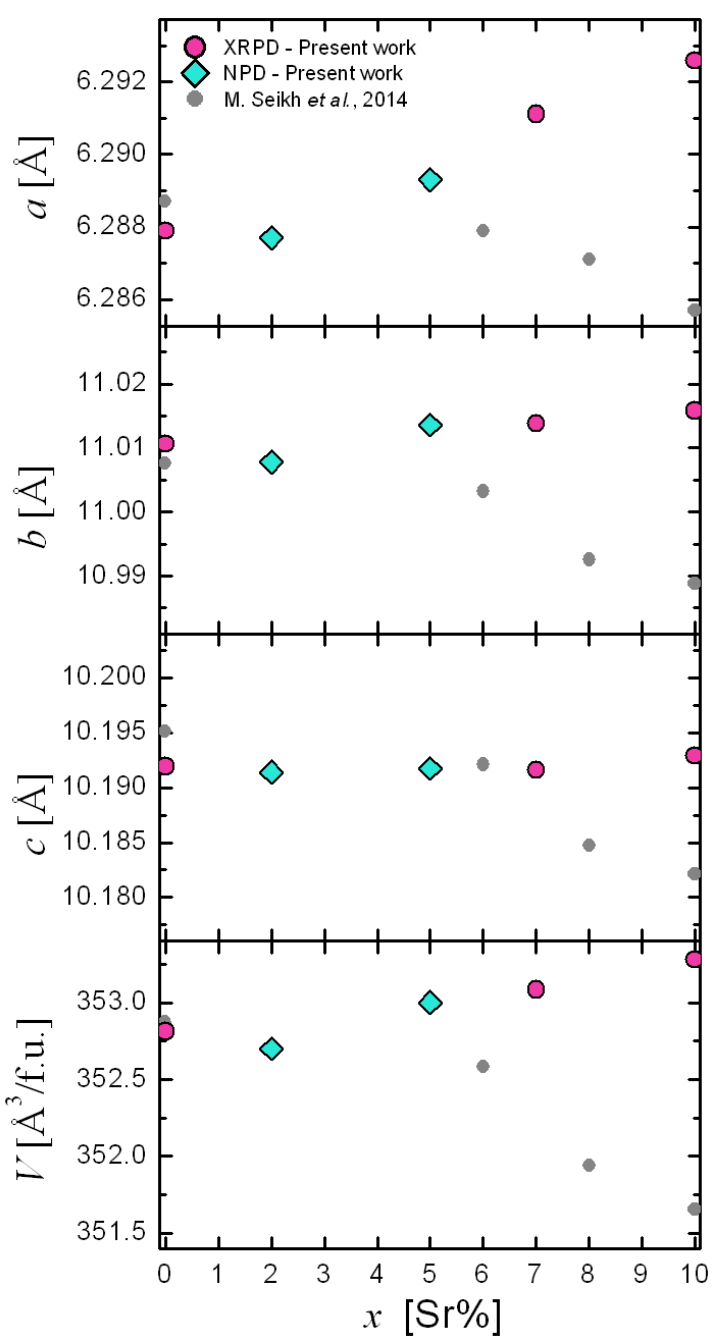

FIG. 2. Evolution of the room-temperature lattice parameters and volume per formula unit in the orthorhombic cell as a function of the Sr substitution $(x)$ in $\left(\mathrm{Ca}_{1-x} \mathrm{Sr}_{x}\right) \mathrm{BaCo}_{4} \mathrm{O}_{7}$. Grey circles represent data for the $\mathrm{Ca}\left(\mathrm{Ba}_{1-x} \mathrm{Sr}_{x}\right) \mathrm{Co}_{4} \mathrm{O}_{7}$ after Ref. $^{25}$. 

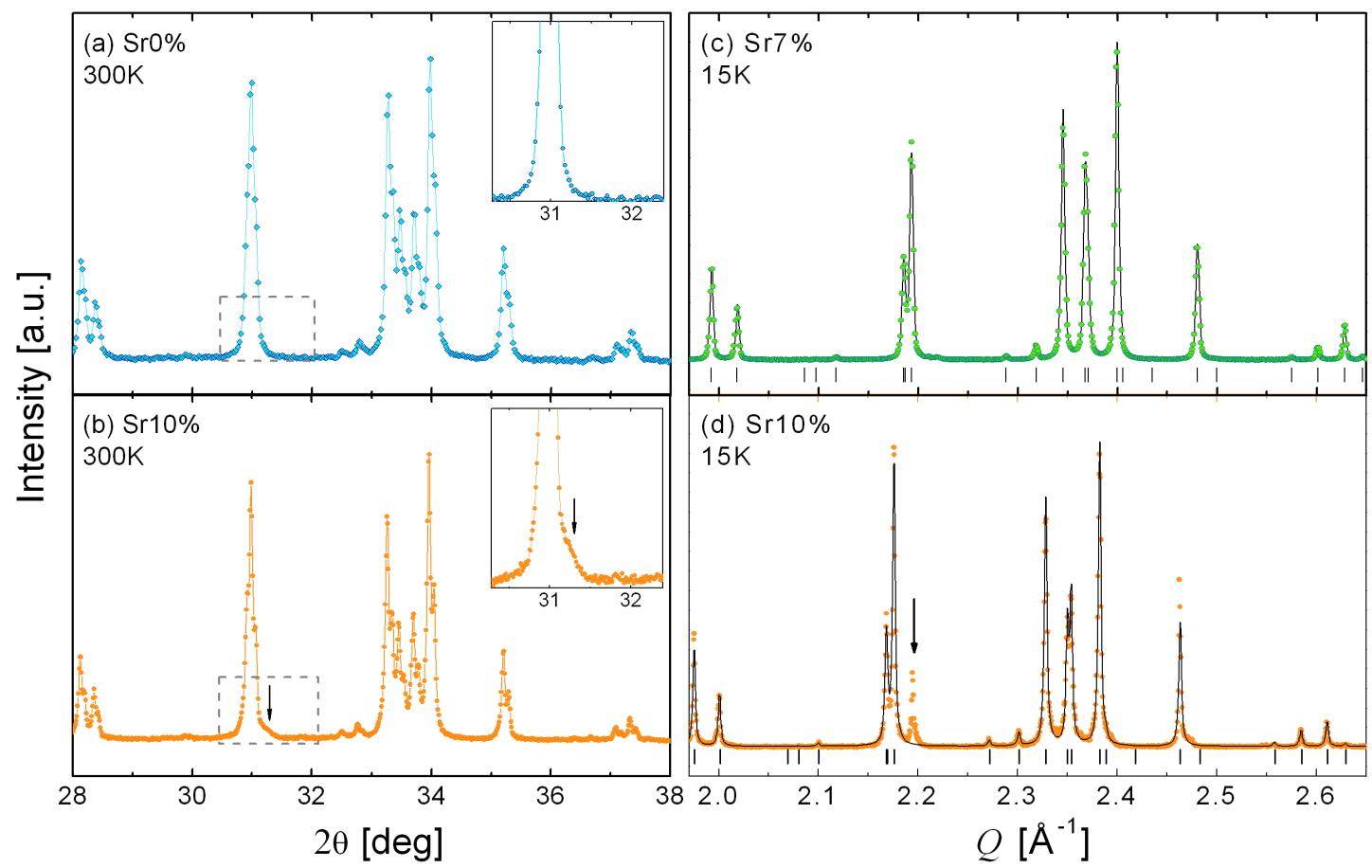

FIG. 3. (a-b) Room temperature laboratory XRPD data for sample $\mathrm{Sr} 0 \%$ (a) and $\mathrm{Sr} 10 \%$ (b) using $\mathrm{Cu} \mathrm{K}-\alpha$ radiation. The insets highlight the region where a reflection from a segregated phase appears for Sr10\%, indicated with an arrow. (c-d) Synchrotron XRPD data at $15 \mathrm{~K}$ for sample Sr7\% (c) and Sr10\% (d) collected at the LNLS synchrotron in Brazil. The solid line represents a Le Bail fit of the majority phase in the $P b n 2_{1}$ space group. The arrow indicates the segregated phase reflection. The LNLS data are plotted as a function of the scattering vector $Q$ to allow the comparison of different wavelengths.

\section{Sr substitution site}

The local structure around Sr was explored by XAS. In Fig. 4 the room temperature crystal structure of $\mathrm{CaBaCo}_{4} \mathrm{O}_{7}$ is sketched. The tetrahedral coordination 

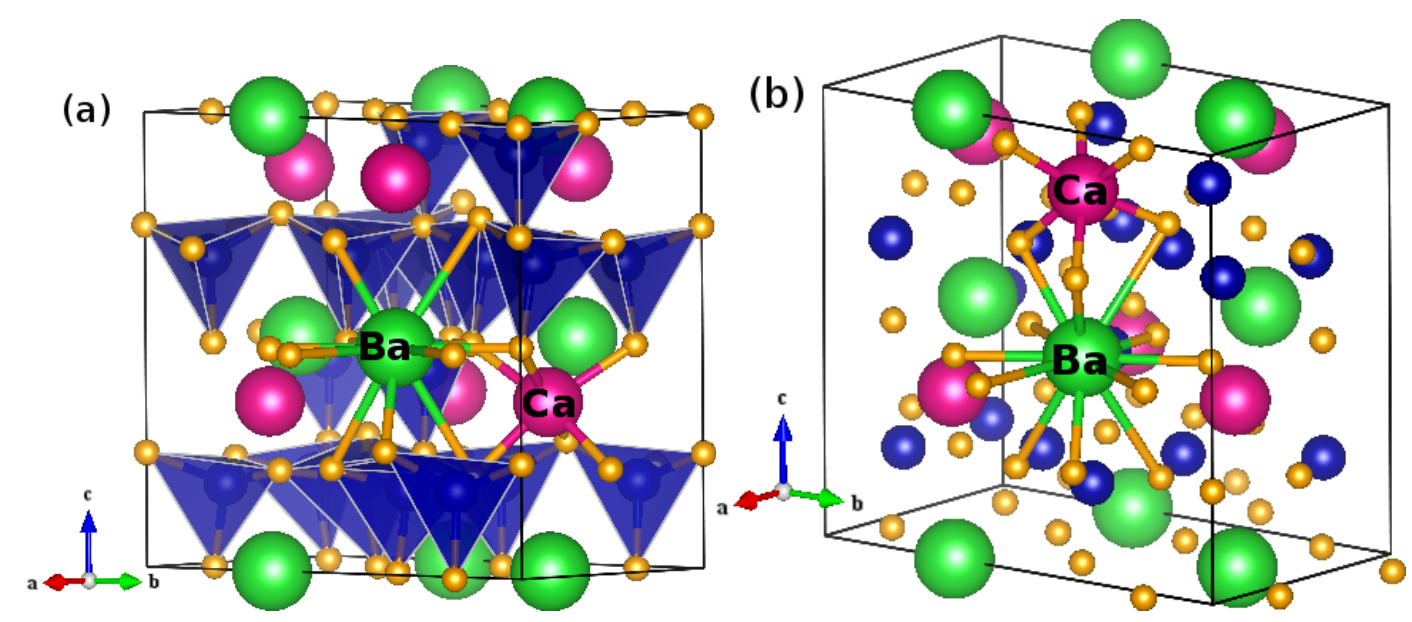

FIG. 4. Crystal structure of $\mathrm{CaBaCo}_{4} \mathrm{O}_{7}$ at room temperature as refined from neutron diffraction data. The oxygen coordination polyhedra around Co are represented in blue (a). (b) First neighbors's shell of Ca and Ba atoms.

of Co atoms is shown in panel (a), whereas the coordination of $\mathrm{Ca}$ and $\mathrm{Ba}$ atoms is highlighted in panel (b). Calcium and Ba cations have very different first coordination shells: Ba atoms are coordinated with 12 oxygen neighbors at an average distance of $3.15 \AA$, and $\mathrm{Ca}$ atoms are coordinated with 6 oxygen atoms at an average distance of $2.34 \AA$, as summarized in Table III. Since the resolution of our XAS data is about $0.05 \AA\left(\delta R=0.2 \cdot \pi / k_{\max }\right.$, where $\left.k_{\max } \sim 13 \AA^{-1}\right)$, the first coordination shell of $\mathrm{Sr}$ at $\mathrm{Ba}$ or Ca sites should differ enough to be distinguished by XAS. For this experiment, we used the Sr7\% sample to obtain the best signal from the Sr K-edge but without reaching the solubility limit.

The Sr K-edge XANES spectra corresponding to the two possible substitution sites, Sr@Ca and Sr@Ba, were modeled using FEFF8.4 calculations, starting from our NPD crystallographic data for the Sr5\% sample. The simulated XANES spectra are shown in Fig. 5, together with the experimental spectrum of sample Sr7\% 


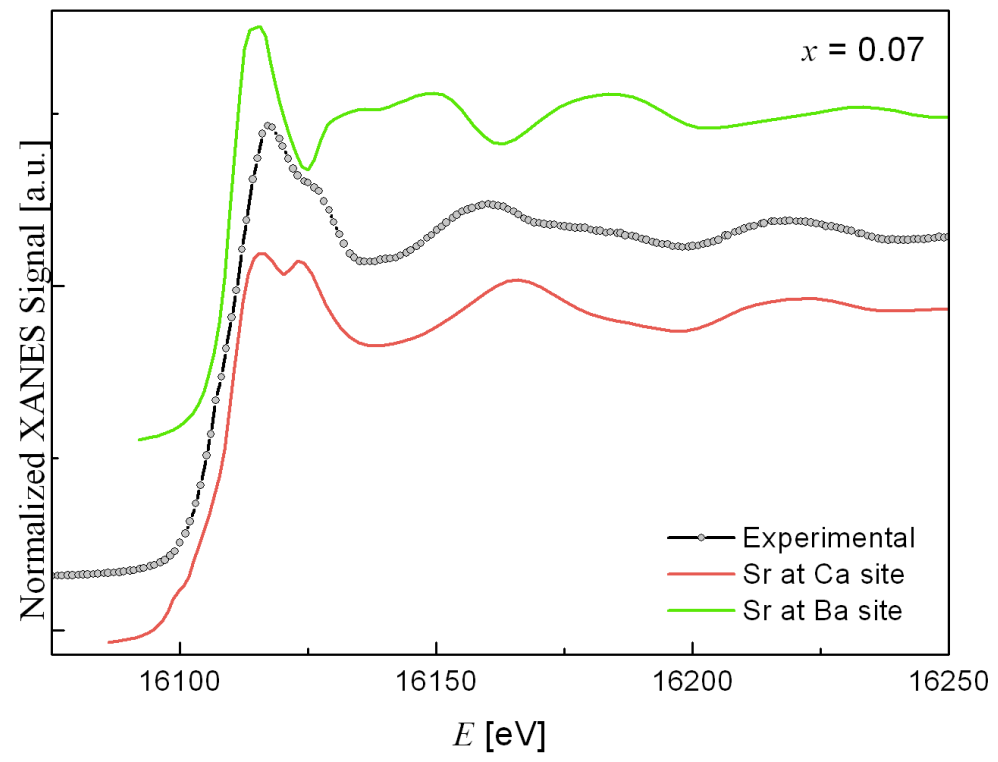

FIG. 5. Simulated Sr K-edge XANES spectra for the Sr@Ca and Sr@Ba models. The experimental spectrum for sample $\mathrm{Sr} 7 \%$ (nominal $\left.\left(\mathrm{Ca}_{0.93} \mathrm{Sr}_{0.07}\right) \mathrm{BaCo}_{4} \mathrm{O}_{7}\right)$ is shown for comparison using symbols. The spectra are vertically shifted for clarity.

which corresponds to the merge of the three collected scans aligned and calibrated to the $\mathrm{Sr} \mathrm{K}$-edge $(16105 \mathrm{eV})$. Although we could not fully reproduce the spectral features, it is evident that there is a better match between the experimental data and the XANES signal calculated for Sr atoms located at the Ca site and that all the features of the experimental spectrum are also qualitative present in the simulated Sr@Ca XANES signal. Therefore, we consider that the latter is the most suitable model to describe our sample, as the XANES signal for the $\mathrm{Sr} @ \mathrm{Ba}$ model shows a much worse agreement.

For the EXAFS analysis, ab-initio amplitude and phase scattering functions were generated using $\mathrm{FEFF}^{19}$ for both Sr@Ba and Sr@Ca models. To account for the lower resolution of XAS data, the first three coordination shells around Sr 

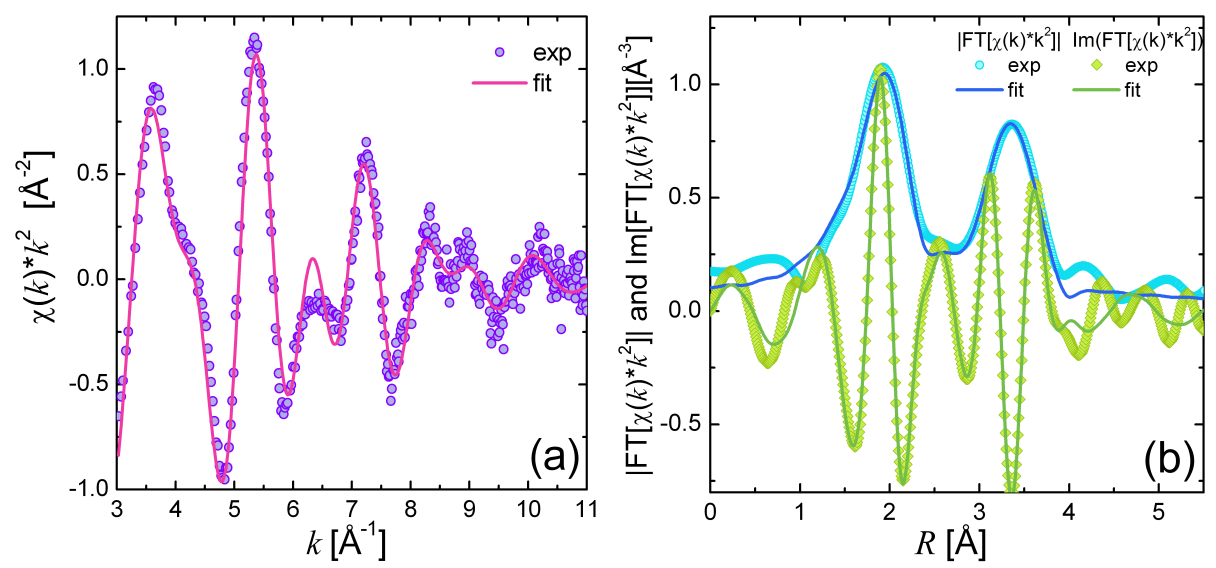

FIG. 6. (a) $k^{2}$-weighted EXAFS signal (symbols) and fit (line) of sample Sr7\% (nominal $\left.\left(\mathrm{Ca}_{0.93} \mathrm{Sr}_{0.07}\right) \mathrm{BaCo}_{4} \mathrm{O}_{7}\right)$. (b) Modulus $(|\mathrm{FT}|)$ and imaginary part (Im) of the Fourier transformed (1-4 A Hanning window) EXAFS signal and fit reported in panel (a). Symbols represent experimental data and solid lines are the fit curves. Fourier transformed data are $k^{2}$-weighted and not corrected for the phase shift.

(Sr-O, Sr-Co and Sr-Ba/Ca) were averaged from the NPD data and are indicated in Table IV. Shell fitting was performed using only the above single-scattering paths. Coordination numbers were kept fixed to the crystallographic structures, while atomic distances, Debye-Waller factors, and the "global" parameters (the many body amplitude reduction factor, $S_{0}^{2}$, and the energy shift, $\Delta \mathrm{E}$ ) were left free to vary during minimizations. The EXAFS signal and fit curve using the Sr@Ca model, together with their Fourier transforms, are shown in Fig. 6.

The structural parameters extracted from the EXAFS fit at the Sr K-edge are reported in Table IV and compared with the structures calculated from the $\mathrm{Sr} @ \mathrm{Ca}$ and Sr@Ba models based on averaged neutron diffraction data. The comparison reported in Table IV shows that the experimental data match well with the model 
where Sr substitutes at the Ca site $(\mathrm{Sr} @ \mathrm{Ca})$. The first coordination shell $(\mathrm{Sr}-\mathrm{O})$ average distance is larger than in the NPD-model of $\mathrm{Sr} @ \mathrm{Ca}$, in agreement with the larger size of $\mathrm{Sr}$ with respect to $\mathrm{Ca}$. The second coordination shell ( $\mathrm{Sr}-\mathrm{Co}$ ) average distance matches with both $\mathrm{Sr} @ \mathrm{Ca}$ and $\mathrm{Sr} @ \mathrm{Ba}$ models, in agreement with the fact that $\mathrm{Sr}$ "sees" the same cobalt environment from both $\mathrm{Ca}$ and $\mathrm{Ba}$ sites. The refinement of the third coordination shell would be conclusive in revealing the substitution site, because Sr would only "see" Ba if located at the Ca site ( $\mathrm{Sr} @ \mathrm{Ca})$ and only $\mathrm{Ca}$ if substituting at the Ba site ( $\mathrm{Sr} @ \mathrm{Ba})$. Both Sr-Ba and Sr-Ca contributions were used to fit the third coordination shell, but, although the match with experimental data improves when using Sr-Ba scattering paths, the results are not conclusive. In fact, even if, in principle, XAS would allow distinguishing between Sr-Ba and Sr-Ca contributions to the third shell, the correlation between parameters dramatically lowers its chemical sensitivity. On the other hand, all attempts to fit the data with the model Sr@Ba led to bad matches with the experimental data and to unphysical values of the $S_{0}^{2}$ parameter. Therefore, despite restrictions in analyzing the third shell by EXAFS, the complete XAS analysis allows to conclude that $\mathrm{Sr}$ sits at the $\mathrm{Ca}$ site in the structure, so we can be confident that the nominal composition is correct and the discussion on the effects of replacing Ca-by-Sr may rely on this result.

\section{MAGNETIC PROPERTIES}

The effects of Sr-substitution on the magnetism of the $\mathrm{CaBaCo}_{4} \mathrm{O}_{7}$ system were studied using magnetometry techniques. For the lowest level of substitution studied here, corresponding to 2 at.\% Sr-for-Ca, we observe a very similar magnetic behaviour as for the parent compound. The comparison between the Sr0\% and Sr2\% samples is presented in Fig. 7. It may be inferred from this figure 


\begin{tabular}{|c|c|c|c|}
\hline \multirow{2}{*}{$\begin{array}{l}\text { photoelectron } \\
\text { path }\end{array}$} & \multicolumn{2}{|c|}{ models } & \multirow{2}{*}{$\begin{array}{l}\text { EXAFS data } \\
\text { Sample Sr7\% }\end{array}$} \\
\hline & $\mathrm{Sr} @ \mathrm{Ca}$ & $\mathrm{Sr} @ \mathrm{Ba}$ & \\
\hline & $\mathrm{CN}$ & $\mathrm{CN}$ & $\mathrm{CN}$ \\
\hline & $\mathrm{R}(\AA)$ & $\mathrm{R}(\AA)$ & $\mathrm{R}(\AA)$ \\
\hline & & & $\sigma^{2}\left(\AA^{2}\right)$ \\
\hline \multirow[t]{3}{*}{$\mathrm{Sr}-\mathrm{O}$} & 6 & 12 & 6 \\
\hline & 2.338 & 3.150 & $2.46(1)$ \\
\hline & & & $0.009(1)$ \\
\hline \multirow[t]{3}{*}{$\mathrm{Sr}-\mathrm{Co}$} & 12 & 12 & 12 \\
\hline & 3.688 & 3.687 & $3.71(1)$ \\
\hline & & & $0.018(2)$ \\
\hline \multirow[t]{3}{*}{$\mathrm{Sr}-\mathrm{Ba}$} & 4 & - & 4 \\
\hline & 3.856 & - & $3.90(5)$ \\
\hline & & & $0.018(6)$ \\
\hline \multirow[t]{5}{*}{$\mathrm{Sr}-\mathrm{Ca}$} & - & 4 & - \\
\hline & - & 3.856 & - \\
\hline & & & $\mathrm{S}_{0}^{2}=0.9(1)$ \\
\hline & & & $\Delta E=1.2(9)$ \\
\hline & & & $\chi_{\nu}^{2}=4.3$ \\
\hline
\end{tabular}

TABLE IV. Comparison between the crystallographic structure around the Sr atom of the Sr@Ca and Sr@Ba models calculated from neutron diffraction data and the results of the EXAFS refinements for sample $\mathrm{Ca}\left(\mathrm{Ba}_{0.93} \mathrm{Sr}_{0.07}\right) \mathrm{Co}_{4} \mathrm{O}_{7}(\mathrm{Sr} 7 \%)$ (CN, coordination numbers; R, atomic distances; $\sigma^{2}$, Debye-Waller factors; $\mathrm{S}_{0}^{2}$, amplitude reduction factor; $\Delta E$, energy shift; $\chi_{\nu}^{2}$, reduced chi-square). The numbers between parentheses represent the error on the last digit. 
that the $\mathrm{Sr} 2 \%$ sample also undergoes a transition to a ferrimagnetic phase with a similar saturation value of magnetization at the lowest temperature and with a $T_{C}$ slightly lower than for the parent compound. The inset in Fig. 7 shows the ac-magnetization $\left(\chi^{\prime}(T)\right)$ for the two samples under different frequencies. For the parent compound, we observe the expected curve for a stoichiometric sample ${ }^{15,24}$, with a unique sharp peak centered at $T_{C}=60 \mathrm{~K}$ which is frequency-independent. No further features at all are observed at lower temperatures, which have been reported to appear in cases where the stoichiometry is not perfect, being $\chi^{\prime}(T)$ one of the most sensitive properties to the presence of new magnetic interactions introduced by doping and/or stoichiometry deviations ${ }^{24,29}$. Despite the similarities with the parent compound, the $\chi^{\prime}(T)$ curve for the $\mathrm{Sr} 2 \%$ sample reveals some indications of additional magnetic interactions in the system. To begin with, the peak corresponding to the ferrimagnetic transition has become less sharp and has shifted to a lower temperature. In addition, a small kink has appeared at $\sim 80 \mathrm{~K}$, which has been repeatedly reported for several kinds of substitutions in the $\mathrm{CaBaCo}_{4} \mathrm{O}_{7}$ system $^{13,24,30,31}$.

More insight into the origin of these features may be derived from the evolution of the magnetization curves upon further substitution with Sr. In Fig. 8 we show the magnetization as a function of temperature for the whole series of Srsubstituted samples, with $0.02 \leq x \leq 0.10$. Note that the data are presented in a logarithmic scale, to stress the dramatic decrease of the net magnetization upon $\mathrm{Sr}$ substitution. It is immediately clear that the feature at $\sim 80 \mathrm{~K}$ is systematic, and gradually evolves into a "plateau" of the magnetization in a temperature range whose lower limit is $x$-dependent. This "plateau" suggests the existence of antiferromagnetic(AFM)-like order in competition with the ferrimagnetic phase, preventing and/or delaying the sharp raise in magnetization at the $T_{C}$.

The similar raise in magnetization below $60 \mathrm{~K}$ for the $\mathrm{Sr} 2 \%$ sample suggests 


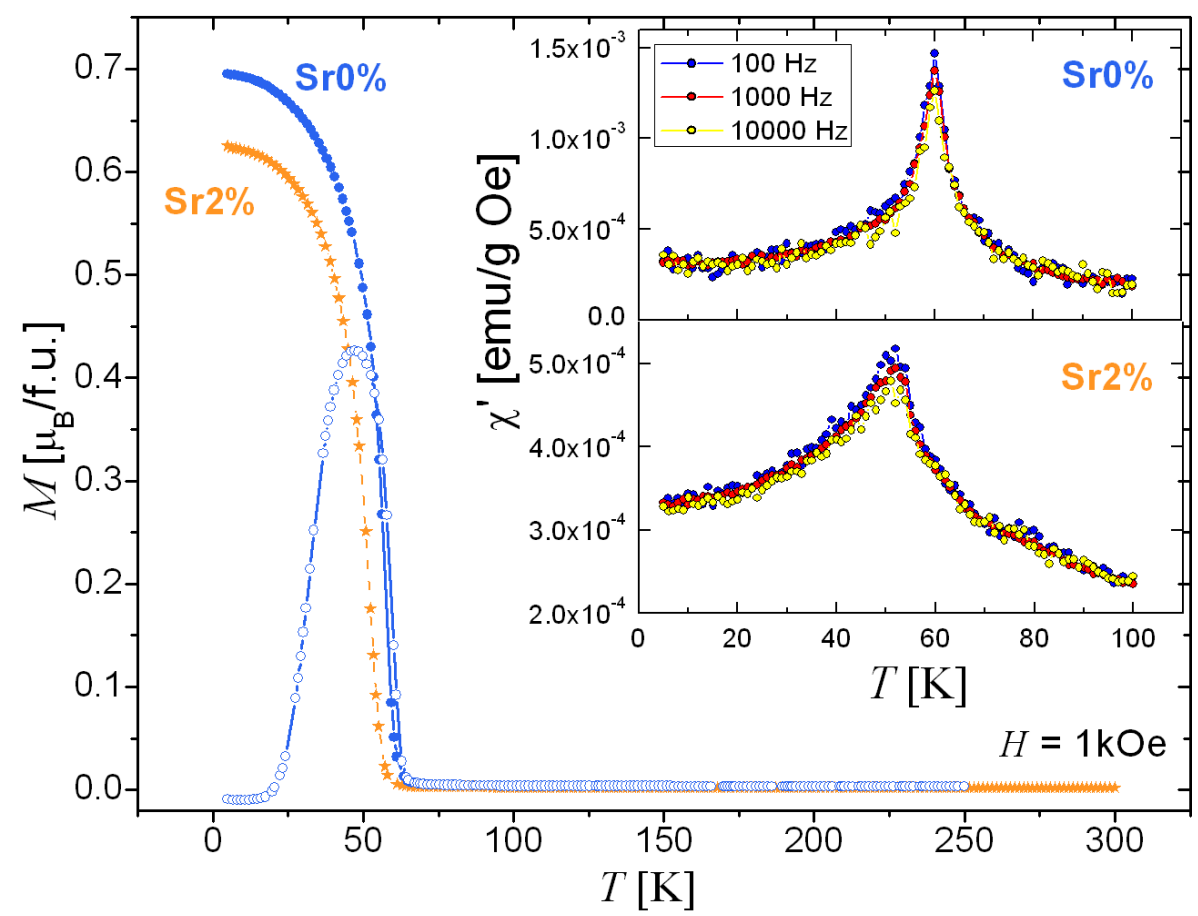

FIG. 7. DC-magnetization as a function of temperature under an applied field of 1000 Oe for $\mathrm{CaBaCo}_{4} \mathrm{O}_{7}$ and $\mathrm{Ca}_{0.98} \mathrm{Sr}_{0.02} \mathrm{BaCo}_{4} \mathrm{O}_{7}$. Open symbols correspond to a zerofield cooled (ZFC) magnetization curve collected on warming, whereas solid symbols correspond to the magnetization collected on cooling under an applied field (FCC). The insets show the temperature dependence of the real part of the ac-susceptibility under an applied field $H_{a c}=10$ Oe and $H_{d c}=0$ Oe measured at different frequencies.

that the ferrimagnetic phase characteristic of the parent compound is preserved. On the other hand, the curve for the Sr5\% sample already indicates that the order has changed dramatically at low temperature, and further substitution enhances this idea. Moreover, the inset in Fig. 8 shows a magnification of the low-temperature region to highlight what we believe are two different transitions characterized by different $T_{C}$ values and saturation magnetizations. We have out- 


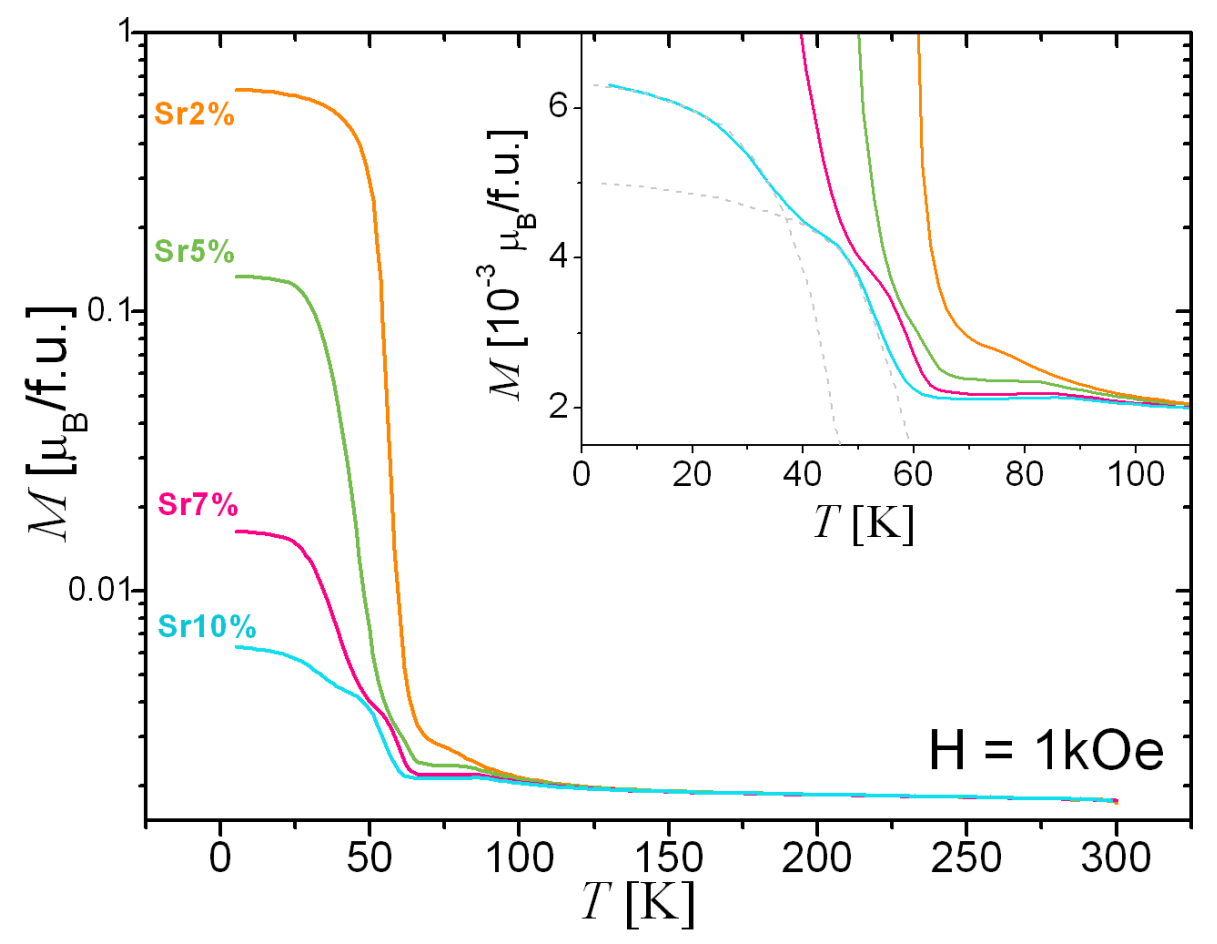

FIG. 8. Magnetization as a function of temperature under an applied field of 1000 Oe for $\mathrm{Ca}_{1-x} \mathrm{Sr}_{x} \mathrm{BaCo}_{4} \mathrm{O}_{7}$ with $x=0.02,0.05,0.07$ and 0.10. Data were collected with a FCC protocol. The inset shows a magnification of the low temperature data in a linear scale. The dashed lines on the Sr10\% curve are only guides to the eye.

lined this features with dashed lines for the Sr10\% sample in the inset. Although the $x=0.10$ sample has a small amount of a segregated perovskite phase, its magnetization behaviour is completely in line with the systematics of Sr-addition, and the evidence of two different transitions at temperatures below the "plateau" is also observed in samples with $x=0.07$ and 0.05 in which there are no segregated phases at all. We would finally like to stress the similarities yet differences with the behavior reported in Ref. ${ }^{29}$ for the Sr-for-Ba substitution. The presence of 
the magnetization "plateau" is clearly a common feature, however below $60 \mathrm{~K}$ the curves do not look similar. The authors propose a scenario of competition between the ferrimagnetic phase of the parent compound and a frustrated magnetic phase with a frequency dependent ac-susceptibility which is not at all compatible with our data. The present magnetization curves at low temperature are rather more similar to those reported for Li-substituted samples ${ }^{31}$ which were reported as a Li-for-Co substitution. It is evident that the variety of transitions suggested by the dc- and ac-magnetization curves deserve a more profound study, which is now in progress and will be reported separately. It is also clear that a deep discussion on the reasons for the impressive effects of substitution must rely on the correct identification of substitution sites in the structure, which is the main objective of the present work.

\section{CONCLUSIONS}

The Ca-based "114" cobaltates have opened up exciting possibilities for the study of magnetoelectricity, magnetic frustrated states, ferrotoroidicity, etc. ${ }^{10-12}$. Cation substitution has proven to trigger even more interesting questions regarding the phase competition in the system, as it deeply affects their magnetism, charge order and transport properties. The accurate knowledge of the substitution site usually remains a standing issue, despite being of crucial importance to understand the magnetic phenomena.

In this work, we have investigated the cationic arrangements in Sr-doped Ca"114" cobaltates combining long-range structural probes, such as neutron and

X-ray diffraction, with short-range chemical selective tools, such as EXAFS and XANES simulations. By applying this multi-technique approach, we demonstrated that Sr can substitute at the Ca site with a solubility limit lying around 
$x \simeq 0.08$. However, in the light of the results by other groups ${ }^{25}$, a Sr-for-Ba substitution might also be possible in this system. It is therefore important to correctly characterize the samples before drawing conclusions on the effects of doping on the magnetic, structural and other properties. The present results support our nominal doping site and constitute the base to analyze the impressive effects of the Sr-for-Ca substitution on the magnetic properties. We observe that a small amount of $\mathrm{Sr}$ addition $(x \simeq 0.02)$ preserves the ferrimagnetic phase of the parent compound although there are indications that other magnetic interactions start to compete in the system. This is firmly established by further increasing the Sr-for-Ca substitution, which results in a decrease of magnetization of two orders of magnitude for a 10 at.\% substitution. An AFM state in a temperature region around $60 \mathrm{~K} \lesssim T \lesssim 80 \mathrm{~K}$ is shown to get stabilized with $\mathrm{Sr}$ content. At lower temperature, the ferrimagnetic phase of the parent compound seems to be replaced by other types of order involving even a third transition which needs to be further studied.

\section{ACKNOWLEDGMENTS}

This work is part of a research project supported by Agencia Nacional de Promoción Científica y Tecnológica (Argentina), under grant PICT-2011-0752, by CONICET under grant PIP 490 2012-2014 and by Universidad Nacional de Cuyo under SeCTyP grant 06-C456. We acknowledge Soleil synchrotron for the beamtime allocation and the MinCyT-Soleil collaboration project for the finantial support. We also acknowledge ILL and its staff for the beamtime allocation and technical assistance as well as the MinCyT of Argentina for funding the trip to perform the experiments. We thank A. Zelaya who helped in the preparation of the samples, V.Tognoli and R. Benavides at Centro Atómico Bariloche and Ana 
Arauzo at Universidad de Zaragoza for their valuable technical support. GA and JC thank the CONICET-CSIC program for scientific visits and grant number MAT2011-27233-C02-02.

\section{REFERENCES}

${ }^{1}$ M. Valldor. Solid State Sciences 4, 7 (2002).

${ }^{2}$ A. P. Ramirez. Annual Review of Materials Science 24, 1 (1994).

${ }^{3}$ C. Lacroix, P. Mendels, and F. Mila. Introduction to Frustrated Magnetism: Materials, Experiments, Theory, volume 164 (Springer, 2011).

${ }^{4}$ O. Parkkima, H. Yamauchi, and M. Karppinen. Chemistry of Materials 25, 4 (2013).

${ }^{5} \mathrm{O}$. Parkkima. $\mathrm{YBaCo}_{4} \mathrm{O}_{7+\delta}$ and $\mathrm{YMnO}_{3+\delta}$ Based Oxygen-Storage Materials. Ph.D. thesis, School on Chemical Technology, Aalto University (2014).

${ }^{6}$ V. Caignaert, V. Pralong, A. Maignan, and B. Raveau. Solid State Communications 149, 11 (2009).

${ }^{7}$ V. Caignaert, V. Pralong, V. Hardy, C. Ritter, and B. Raveau. Physical Review B 81 (2010).

${ }^{8}$ Z. Qu, L. Ling, L. Zhang, L. Pi, and Y. Zhang. Solid State Communications 151, 13 (2011).

${ }^{9}$ H. Iwamoto, M. Ehara, M. Akaki, and H. Kuwahara. In Journal of Physics: Conference Series, volume 400, p. 032031 (IOP Publishing, 2012).

${ }^{10}$ V. Caignaert, A. Maignan, K. Singh, C. Simon, V. Pralong, B. Raveau, J. F. Mitchell, H. Zheng, A. Huq, and L. Chapon. Physical Review B 88, 17 (2013).

${ }^{11}$ K. Singh, V. Caignaert, L. Chapon, V. Pralong, B. Raveau, and A. Maignan. Physical Review B 86, 2 (2012).

${ }^{12}$ R. Johnson, K. Cao, F. Giustino, and P. Radaelli. Physical Review B 90, 4 
(2014).

${ }^{13}$ T. Sarkar, M. M. Seikh, V. Pralong, V. Caignaert, and B. Raveau. Journal of Materials Chemistry 22, 34 (2012).

${ }^{14}$ M. Seikh, T. Sarkar, V. Pralong, V. Caignaert, and B. Raveau. Physical Review B 86, 18 (2012).

${ }^{15}$ Y. Zou, Z. Qu, L. Zhang, W. Ning, L. Ling, L. Pi, and Y. Zhang. Journal of Alloys and Compounds 576 (2013).

${ }^{16}$ G. Aurelio, F. Bardelli, R. J. Prado, R. Sanchez, M. Saleta, and G. Garbarino. Chemistry of Materials (2013).

${ }^{17}$ LAMP, the Large Array Manipulation Program. http://www.ill.eu/data_treat/lamp/the-lamp-book/.

${ }^{18}$ J. Rodríguez-Carvajal. In Abstracts of the Satellite Meeting on Powder Diffraction of the XV Congress of the IUCr, Toulouse, France, p. 127 (1990).

${ }^{19}$ A. Ankudinov, B. Ravel, J. Rehr, and S. Conradson. Physical Review B 58, 12 (1998).

${ }^{20}$ L. Hedin and B. I. Lundqvist. Journal of Physics C: Solid state physics 4, 14 (1971).

${ }^{21}$ J. Rehr and R. Albers. Reviews of Modern Physics 72, 3 (2000).

${ }^{22}$ C. Meneghini, F. Bardelli, and S. Mobilio. Nuclear Instruments and Methods in Physics Research Section B: Beam Interactions with Materials and Atoms 285 (2012).

${ }^{23}$ Kalvados, software for crystal structure and powder diffraction. http://www.fzu.cz/ knizek/kalvados/index.html.

${ }^{24}$ M. M. Seikh, V. Caignaert, E. Suard, K. P. Meher, A. Maignan, and B. Raveau. Journal of Applied Physics 116, 24 (2014).

${ }^{25}$ M. Seikh, V. Pralong, V. Caignaert, B. Raveau, et al. Zeitschrift für anorganische und allgemeine Chemie 640, 6 (2014). 
${ }^{26}$ The full report of a thermodiffraction experiment performed at LNLS in Campinas, Brazil, is beyond the scope of the present work and will be published separately. We show here only a selected area of the $15 \mathrm{~K}$ data for two samples which nicely support the presence of the impurity phase in the Sr10\% sample.

${ }^{27}$ K. Yamaura, H. Zandbergen, K. Abe, and R. Cava. Journal of Solid State Chemistry 146, 1 (1999).

${ }^{28}$ C. de la Calle, J. A. Alonso, and M. T. Fernandez-Diaz. Zeitschrift fur Naturforschung B 63, 6 (2008).

${ }^{29}$ M. M. Seikh, V. Caignaert, V. Pralong, and B. Raveau. Journal of Physics and Chemistry of Solids 75, 1 (2014).

${ }^{30}$ T. Sarkar, M. Motin Seikh, V. Pralong, V. Caignaert, and B. Raveau. Applied Physics Letters 100, 23 (2012).

${ }^{31}$ M. M. Seikh, T. Sarkar, V. Pralong, V. Caignaert, and B. Raveau. Journal of Applied Physics 113, 5 (2013). 